\title{
An Elastic Micropolar Mixture Theory for Predicting Elastic Properties of Cellular Materials
}

\author{
S. Elangovan \\ Department of Mechanical Engineering - Engineering Mechanics \\ Michigan Technological University \\ 931 R.L. Smith Building \\ Houghton, Michigan 49931 \\ B.S. Altan \\ Tiger Strategy LLC \\ 504 West Edwards Avenue \\ Houghton, Michigan 49931 \\ G.M. Odegard* \\ Department of Mechanical Engineering - Engineering Mechanics \\ Michigan Technological University \\ 930 R.L. Smith Building \\ Houghton, Michigan 49931
}

\begin{abstract}
An efficient modeling approach is established to predict the elastic response of cellular materials with distributions of cell geometries. The approach does not require complex and timeconsuming computational techniques usually associated with modeling such materials. Unlike most current analytical techniques, the modeling approach directly accounts for the cellular material microstructure. The approach combines micropolar elasticity theory and elastic mixture theory to predict elastic response of cellular materials to a wide range of loading conditions. The modeling approach is applied to the two-dimensional balsa wood material. Predicted properties are in good agreement with experimentally-determined properties, which emphasizes the model's potential to predict the elastic response of other cellular solids, such as open cell and closed cell foams.
\end{abstract}

Keywords: Couple stress theory, Gradient theory, Honeycombs, Cosserat Elasticity, Lattice structures, Frames

\footnotetext{
* Corresponding author Tel.: (906)487-2329, Fax:(906)487-2822, email: gmodegar@mtu.edu
} 


\section{NOMENCLATURE}

$a_{k}^{(n)}$

$a_{k l}^{(n)}$

$A_{k l}^{(n)}, A_{k l m n}^{(n)}$

$A^{(n)}$

$a_{k l}$

$b_{k l}^{(n)}$

$B_{k l}^{(n)}, B_{k l m n}^{(n)}$

$b_{k l}$

$C_{0}$

$C_{k l m n}^{(n)}$

$D$

$E_{\text {strut }}^{(n)}$

$E^{(n)}$

E

$f_{k}^{(n)}$

$f^{(n)}$

$G_{\text {strut }}^{(n)}$

$G^{(n)}$

G

$h^{(n)}$

$h$

I

$I^{(n)}$

$j^{(n)}$

$J$

K

$l_{i}^{(n)}$

$l^{(n)}$

$m_{k l}^{(n)}$

$\hat{m}_{k}^{(n)}$

$m_{k l}$

$\hat{p}_{k}^{(n)}$

$q_{k}^{(n)}$
Acceleration vector component of the $n^{\text {th }}$ constituent

Material derivative of $\varepsilon_{k l}^{(n)}$

Material moduli of the $n^{\text {th }}$ constituent

Cross-sectional area of the struts of the $n^{\text {th }}$ constituent

Material derivative of $\varepsilon_{k l}$

Material derivative of $\gamma_{k l}^{(n)}$

Material moduli of the $n^{\text {th }}$ constituent

Material derivative of $\gamma_{k l}$

Constant relating temperature and free energy in the natural state

Material moduli of the $n^{\text {th }}$ constituent

Set of all dependent variables

Young's modulus of the strut material of the $n^{\text {th }}$ constituent

Young's modulus of the equivalent continuum of the $n^{\text {th }}$ constituent

Young's modulus of the mixture

Body force density vector components of the $n^{\text {th }}$ constituent

Volume fraction of the $n^{\text {th }}$ constituent

Shear modulus of the material of the struts of the $n^{\text {th }}$ constituent

Shear modulus of the equivalent continuum of the $n^{\text {th }}$ constituent

Shear modulus of the mixture

Internal energy source density of the $n^{\text {th }}$ constituent

Internal energy source density of the mixture

Set of all independent variables

Moment of inertia of the struts of the $n^{\text {th }}$ constituent

Microinertia density of the $n^{\text {th }}$ constituent

Set of all thermodynamic fluxes

Classical Fourier constant

Body couple density vector components of the $n^{\text {th }}$ constituent

Length of the struts of the $n^{\text {th }}$ constituent

Couple stress tensor components of the $n^{\text {th }}$ constituent

Internal couple vector components exerted onto $n^{\text {th }}$ constituent by the other constituent

Couple stress tensor components of the mixture

Internal force density vector components exerted onto $n^{\text {th }}$ constituent by the other constituent

Heat flux vector components of the $n^{\text {th }}$ constituent 


\begin{tabular}{|c|c|}
\hline$q_{k}$ & Heat flux vector components of the mixture \\
\hline$Q^{(n)}$ & Grid structural parameter \\
\hline $\mathcal{R}^{(n)}$ & Region occupied by the $n^{\text {th }}$ constituent in the mixture \\
\hline$R^{(n)}$ & Grid structural parameter \\
\hline $\begin{array}{l}S_{0} \\
S^{(n)}\end{array}$ & Free energy in the natural state \\
\hline $\begin{array}{l}s^{(n)} \\
S^{(n)}\end{array}$ & \\
\hline$S^{(n)}$ & Grid structural parameter \\
\hline$t$ & Time at the end of constituent motion \\
\hline$t_{k l}^{(n)}$ & Stress tensor components of the $n^{\text {th }}$ constituent \\
\hline$T$ & Change in temperature from ambient temperature \\
\hline$T_{0}$ & Ambient temperature \\
\hline$T^{(n)}$ & Grid structural parameter \\
\hline$t_{k l}$ & Stress tensor components of the mixture \\
\hline$u_{k}^{(n)}$ & Displacement vector components of the $n^{\text {th }}$ constituent \\
\hline$u_{k}$ & Displacement vector components of the mixture \\
\hline$v_{k}^{(n)}$ & Velocity vector components of the $n^{\text {th }}$ constituent \\
\hline$v_{i}$ & Velocity vector components of the mixture \\
\hline$v_{k}^{(12)}$ & Relative velocity vector components \\
\hline$X_{k}^{(n)}$ & Reference position vector components of the $n^{\text {th }}$ constituent \\
\hline$x_{k}^{(n)}$ & Spatial position vector components of the $n^{\text {th }}$ constituent \\
\hline$Y$ & Set of all thermodynamic forces \\
\hline$\alpha^{(n)}$ & Micropolar elastic constant of the $n^{\text {th }}$ constituent \\
\hline$\beta_{0}^{(n)}$ & Thermal expansion constant of the $n^{\text {th }}$ constituent \\
\hline$\beta^{(n)}$ & Micropolar elastic constant of the $n^{\text {th }}$ constituent \\
\hline$\chi_{k}^{(n)}$ & Motion equation components of the $n^{\text {th }}$ constituent \\
\hline$\delta_{i j}$ & Kronecker delta \\
\hline$\varepsilon_{k l}^{(n)}$ & Linear strain tensor components of the $n^{\text {th }}$ constituent \\
\hline $\begin{array}{l}\varepsilon_{i j k} \\
\varepsilon^{(n)}\end{array}$ & $\begin{array}{l}\text { Permutation tensor components } \\
\text { Internal energy density of the } n^{\text {th }} \text { constituent }\end{array}$ \\
\hline$\hat{\varepsilon}^{(n)}$ & Energy transferred to $n^{\text {th }}$ constituent from the other constituent \\
\hline$\varepsilon$ & Internal energy density of the mixture \\
\hline$\varepsilon_{k l}$ & Strain tensor components of the mixture \\
\hline$\phi_{i}^{(n)}$ & Microrotation vector components of the $n^{\text {th }}$ constituent \\
\hline$\Phi$ & Dissipation potential \\
\hline$\phi_{i}$ & Microrotation vector components of the mixture \\
\hline$\gamma_{k l}^{(n)}$ & Linear microrotation gradient tensor components of the $n^{\text {th }}$ constituent \\
\hline$\gamma^{(n)}$ & Micropolar elastic constant of the $n^{\text {th }}$ constituent \\
\hline$\gamma_{k l}$ & Linear microrotation gradient tensor components of the mixture \\
\hline
\end{tabular}




$\begin{array}{ll}\eta & \text { Entropy density of the mixture } \\ \eta_{0} & \text { Entropy density of the mixture in the natural state } \\ \kappa^{(n)} & \text { Micropolar elastic constant of the } n^{\text {th }} \text { constituent } \\ \lambda^{(n)} & \text { Micropolar elastic constant of the } n^{\text {th }} \text { constituent } \\ \mu^{(n)} & \text { Micropolar elastic constant of the } n^{\text {th }} \text { constituent } \\ v^{(n)} & \text { Poisson's ratio of the equivalent continuum of the } n^{\text {th }} \text { constituent } \\ v & \text { Poisson's ratio of the mixture } \\ \theta & \text { Absolute temperature of the constituents and the mixture } \\ \rho^{(n)} & \text { Mass density of the mixture } \\ \rho^{(n)} & \text { Mass density of the } n^{\text {th }} \text { constituent } \\ \rho_{r e l}^{(n)} & \text { Relative density of the grid of the } n^{\text {th }} \text { constituent } \\ v_{i}^{(n)} & \text { Microgyration vector components of the } n^{\text {th }} \text { constituent } \\ v_{k}^{(12)} & \text { Relative microgyration vector components } \\ \varpi & \text { Momentum generation coefficient due to difference in gyrations } \\ \xi & \text { Momentum generation coefficient due to velocity difference } \\ \psi & \text { Free energy density of the mixture } \\ \zeta & \text { Heat generation for unit change in velocity }\end{array}$

\section{INTRODUCTION}

Cellular materials have a lattice architecture that in many cases results in high specific stiffness, specific strength, and good thermal insulation properties relative to many engineering materials. Cellular materials have been used in many structural engineering applications, including the core material in composite sandwich panels (Gibson and Ashby, 1999). The design and implementation of cellular materials relies on accurate and efficient models to relate the lattice microstructure to the bulk mechanical properties.

Cellular materials consist of a complex interconnected framework of either material struts only (open cell foams) or material struts and cell face membranes (closed cell foams) that yields a porous- or a closed-cellular material, respectively. For example, the open cell lattice of the polyurethane foam shown in Figure 1 has pores and material struts with a range of cell sizes and shapes distributed spatially in the material. This microstructural complexity poses significant problems for accurately modeling the mechanical behavior of cellular materials. Two modeling approaches that have been used to predict the mechanical response of cellular materials are finite element analysis (FEA) and analytical techniques. Numerous studies have been performed to predict the mechanical response of cellular materials using FEA techniques (Zhu et al., 2000; Roberts and Garboczi, 2002; Kanaun and Tkachenko, 2006; Li et al., 2006; Yoo and Jasiuk, 2006). While this approach has the ability to accurately predict mechanical behavior for a wide range of cellular microstructures, the model building and solution procedures can be timeconsuming and expensive, making FEA techniques prohibitive where efficient material design is necessary. Analytical approaches have been developed that are much more efficient than FEA approaches (Gibson and Ashby, 1999). Although these methods are simple and efficient, they 
often cannot directly incorporate the microstructural details on non-periodic geometries that are found in a majority of cellular materials without resorting to the use of empirical data. Therefore, there is a need to establish an efficient and accurate modeling approach to predict the mechanical response of cellular materials as a function of the random material microstructure.

The objective of this study is to establish a linear-elastic constitutive modeling approach for cellular materials that accounts for distributions of cell size and geometry. The modeling approach consists of two steps. First, two individual lattice geometries with periodic microstructures are modeled as effective micropolar continua. The micropolar elasticity theory is used to enable the effective continua to represent the lattice geometry at the microstructural and bulk-level length scales. An example of this first step is shown in Figure 2 for two 2-dimensional triangular grids. In the second step the two effective micropolar continua are combined via mixture theory to create a micropolar continuum that effectively models a cellular solid with a distribution of cell geometries, as shown in Figure 2. The proposed approach does not directly model a representative volume element of the material microstructure. The effective behavior of the homogenized micropolar mixture represents the mechanical behavior of the conceptual combined grid shown in Figure 2. The conceptual combined grid is composed of individual periodic grids; however, the periodicity of the conceptual combined grid is dictated by the overlay of the individual grids. Therefore, the modeling approach is capable of modeling cellular materials with little or no periodicity.

First, the general aspects of the micropolar elasticity theory and the elastic mixture theory are detailed, followed by a discussion of the kinematics of the proposed micropolar mixture framework. This is followed by a discussion of the balance laws for a micropolar mixture of two solid constituents. A brief development of the constitutive theory for a mixture of two micropolar solids is subsequently presented. Finally, the application of the proposed modeling approach to a two-dimensional cellular solid is presented.

\section{BACKGROUND}

A brief discussion of micropolar elasticity and mixture theory is presented in this section to facilitate the development of the proposed theory in the proceeding sections.

\subsection{Micropolar Elasticity}

In the theory of micropolar elasticity (Eringen, 1999), the points in a material continuum have six degrees of freedom: three translational components of the classical theory and three rotation components about the coordinate axes. Although the theory of micropolar elasticity has been applied to many fields of engineering, one of its primary uses is to provide a mathematical foundation to describe the mechanical behavior of lattice structures. The micropolar theory is necessary in the analysis of lattice structures because rotations at the strut joints play a significant role in influencing the overall behavior of the lattice at relatively small length scales. For example, a micropolar beam model was developed (Noor and Nemeth, 1980b) for four different types of planar lattice grids with rigid joints. In a subsequent study (Noor and Nemeth, 1980a), the model was extended to model three-dimensional lattice structures. An energy 
approach considering in-plane displacements as well as joint rotations was used (Sun and Yang, 1973) to estimate the dynamic characteristic of a two-dimensional square grid.

\subsection{Mixture Theory}

Motivated by Maxwell's kinetic theory of gases, Truesdell and Toupin (Truesdell and Toupin, 1960) presented an axiomatic mixture theory for interacting continua based on the premise that each point of space is simultaneously occupied by all constituents of the mixture. It has been used with profound success to model the mechanical behavior of composite materials (Bedford and Stern, 1972; Stern and Bedford, 1972; Hegemier et al., 1973; Nayfeh and Gurtman, 1974; McNiven and Mengi, 1979a; McNiven and Mengi, 1979b; McNiven and Mengi, 1979c). It is also assumed that the interactions between the constituents of a mixture are accounted for as interaction forces in the appropriate field equations. The mixture theory concept has been used to model the three-dimensional mechanical behavior of a binary mixture (Bedford and Stern, 1972). In a subsequent study (Stern and Bedford, 1972), the three-dimensional model predicted wave propagation phenomena in a composite material. It was concluded that in order to accurately predict wave propagation properties in a composite material, the material microstructure must be considered, which can be done with considerable ease by using the elastic mixture theory. An alternative version of the mixture theory was developed (Hegemier et al., 1973) for modeling the wave propagation in laminated and unidirectional fibrous composites. The theory was used to determine the distribution of displacements and stresses within individual constituents. This theory was subsequently applied (Nayfeh and Gurtman, 1974) for both transversely and horizontally polarized shear waves propagating in the plane of the laminate. A mixture theory was developed (McNiven and Mengi, 1979a) for two-phase materials which was implemented (McNiven and Mengi, 1979b; McNiven and Mengi, 1979c) to study the wave propagation in periodically-structured composites.

\section{KINEMATICS}

The following presentation of the kinematic theory of a binary mixture of micropolar solids is similar to that developed for a binary mixture of a solid and fluid previously (Eringen, 2003). Consider regions of two constituents of a mixture $\mathcal{R}^{(1)}$ and $\mathcal{R}^{(2)}$, which simultaneously occupy a common volume in the reference configuration (Figure 3). A material point of the $n^{\text {th }}$ constituent in the reference configuration has a position denoted by $\mathbf{X}^{(\mathrm{n})}$. After a time $t$, due to a motion denoted by the mapping $\chi^{(n)}$, the position of the point of the $n^{\text {th }}$ constituent in the spatial configuration is given by the motion equation

$$
\mathbf{x}^{(n)}=\chi^{(n)}\left(\mathbf{X}^{(n)}, t\right)
$$

The corresponding velocity and acceleration vector components at time $t$ are given by, respectively,

$$
\begin{aligned}
& v_{k}^{(n)}=\dot{x}_{k}^{(n)} \\
& a_{k}^{(n)}=\ddot{x}_{k}^{(n)}
\end{aligned}
$$


where the superposed dot denotes the usual material time derivative. The velocity vector components of the mixture are given by

$$
v_{k}=\frac{1}{\rho}\left[\rho^{(1)} v_{k}^{(1)}+\rho^{(2)} v_{k}^{(2)}\right]
$$

where $\rho$ and $\rho^{(n)}$ are the densities of the mixture and the $n^{\text {th }}$ constituent, respectively, and are related by

$$
\rho(x, t)=\rho^{(1)}(x, t)+\rho^{(2)}(x, t)
$$

Because the cellular material is modeled as an equivalent, homogeneous continuum (Figures 2 and 3) the density is not necessarily a function of position even though the density is a function of position in an actual cellular solid microstructure. However, the density is generally modeled as a function of position in Equation (5). The linear strain tensor components and the linear microrotation gradient tensor components of the $n^{\text {th }}$ constituent are given by, respectively,

$$
\begin{gathered}
\varepsilon_{k l}^{(n)}=u_{l, k}^{(n)}+\varepsilon_{l k m} \phi_{m}^{(n)} \\
\gamma_{k l}^{(n)}=\phi_{k, l}^{(n)}
\end{gathered}
$$

where $u_{k}^{(n)}$ and $\phi_{k}^{(n)}$ are the displacement and rotation vector components of a point of the $n^{\text {th }}$ constituent and $\varepsilon_{l k m}$ is the permutation symbol. In Equations (6) and (7), and throughout this paper, the usual summation and partial differentiation conventions are used. For the linear theory, the microgyration vector of the $n^{\text {th }}$ constituent is defined as (Eringen, 1999)

$$
v_{k}^{(n)}=\dot{\phi}_{k}^{(n)}
$$

The material derivatives of (6) and (7) are denoted by

$$
\begin{aligned}
& \dot{\varepsilon}_{k l}^{(n)}=a_{k l}^{(n)} \\
& \dot{\gamma}_{k l}^{(n)}=b_{k l}^{(n)}
\end{aligned}
$$

The motion of the material points of the mixture is influenced by forces and thermodynamic conditions that the mixture is exposed to. The corresponding balance laws are described in the next section.

\section{BALANCE LAWS}

If it is assumed that microinertia is constant and isotropic, then the remaining balance laws of mass, linear momentum, angular momentum, and energy provide field equations which dictate 
the kinetic response of the micropolar mixture. Each balance law is individually addressed in this section. The balance laws are similar to those previously proposed by Eringen (Eringen, 2003).

\subsection{Balance of Mass}

The balance of mass for the $n^{\text {th }}$ constituent is given by

$$
\dot{\rho}^{(n)}+\rho^{(n)} v_{k, k}^{(n)}=0
$$

Summing over the two constituents and using Equations (4) and (5), the balance of mass of the mixture is

$$
\dot{\rho}+\rho v_{k, k}=0
$$

\subsection{Balance of Linear Momentum}

The balance of linear momentum for the $n^{\text {th }}$ constituent is

$$
\rho^{(n)} \ddot{x}_{l}^{(n)}=t_{k l, k}^{(n)}+f_{l}^{(n)}+\hat{p}_{l}^{(n)}
$$

where $\ddot{x}_{l}^{(n)}$ is the acceleration of the $n^{\text {th }}$ constituent, $t_{k l}^{(n)}$ are the components of the stress tensor, $f_{l}^{(n)}$ are the components of the body force density vector, and $\hat{p}_{l}^{(n)}$ are the components of the interaction force density vector which represents force exerted on the $n^{\text {th }}$ constituent due to the interaction with the other constituent (terms that represent constituent interactions have the superposed caret). Summing over the two constituents produces the linear momentum balance of the mixture

$$
\rho^{(1)} \ddot{x}_{l}^{(1)}+\rho^{(2)} \ddot{x}_{l}^{(2)}=t_{k l, k}^{(1)}+t_{k l, k}^{(2)}+f_{l}^{(1)}+f_{l}^{(2)}+\hat{p}_{l}^{(1)}+\hat{p}_{l}^{(2)}
$$

If it is assumed that linear momentum of the mixture is balanced solely by the stresses and body force densities in the constituents then Equation (13) becomes

$$
\hat{p}_{k}^{(1)}+\hat{p}_{k}^{(2)}=0
$$

Equation (14) indicates that the two interaction force density vectors are of equal magnitude and opposite sign.

\subsection{Balance of Angular Momentum}

The balance of angular momentum is given by

$$
\rho^{(n)} j^{(n)} \dot{v}_{l}^{(n)}=m_{k l, k}^{(n)}+\varepsilon_{l m n} t_{m n}^{(n)}+l_{l}^{(n)}+\hat{m}_{l}^{(n)}
$$


where $j^{(n)}$ is the microinertia density (resistance to microrotation), $v_{l}^{(n)}$ is the microgyration (microrotation rate), $m_{k l}^{(n)}$ are the components of the couple stress tensor, $l_{l}^{(n)}$ are the components of the body couple density vector, and $\hat{m}_{l}^{(n)}$ is the components of the interaction couple exerted on the $n^{\text {th }}$ constituent due to interaction with the other constituent. Summing over the two constituents, Equation (15) becomes

$$
\rho^{(1)} j^{(1)} \dot{v}_{l}^{(1)}+\rho^{(2)} j^{(2)} \dot{v}_{l}^{(2)}=m_{k l, k}^{(1)}+m_{k l, k}^{(2)}+\varepsilon_{l m n} t_{m n}^{(1)}+\varepsilon_{l m n} t_{m n}^{(2)}+l_{l}^{(1)}+l_{l}^{(2)}+\hat{m}_{l}^{(1)}+\hat{m}_{l}^{(2)}
$$

If it is assumed that the angular momentum of the mixture is balanced by $m_{k l, k}^{(n)}, t_{m n}^{(n)}$, and $l_{l}^{(n)}$, then Equation (16) reveals

$$
\hat{m}_{l}^{(1)}+\hat{m}_{l}^{(2)}=0
$$

Therefore, the interaction couple vectors have equal magnitudes and opposite signs.

\subsection{Balance of Energy}

The conservation of energy for the $n^{\text {th }}$ constituent is

$$
\dot{\varepsilon}^{(n)}=t_{k l}^{(n)} a_{k l}^{(n)}+m_{k l}^{(n)} b_{l k}^{(n)}+q_{k, k}^{(n)}+h^{(n)}+\hat{\varepsilon}^{(n)}
$$

where $\varepsilon^{(n)}$ is the internal energy density of the $n^{\text {th }}$ constituent, $q_{k}^{(n)}$ is the heat flux vector, $h^{(n)}$ is the energy source density, and $\hat{\varepsilon}^{(n)}$ denotes the transfer of energy density to the $n^{\text {th }}$ constituent from the other constituent. It has been shown (Eringen, 2003) that by summing Equation (18) over the two constituents and utilizing field equations (14) and (17) the following relationship is established

$$
\dot{\varepsilon}=q_{k, k}+h+t_{k l}^{(1)} a_{k l}^{(1)}+t_{k l}^{(2)} a_{k l}^{(2)}+m_{k l}^{(1)} b_{l k}^{(1)}+m_{k l}^{(2)} b_{l k}^{(2)}-\hat{p}_{k}^{(1)} v_{k}^{(12)}-\hat{m}_{k}^{(1)} v_{k}^{(12)}
$$

where $\varepsilon, h$ and $q_{k}$ are the components of the internal energy density, energy source density and heat flux vector of the mixture, respectively, $v_{k}^{(12)}$ and $v_{k}^{(12)}$ are the components of the relative velocity and relative microgyration vectors, respectively, given by

$$
\begin{aligned}
& v_{k}^{(12)}=v_{k}^{(1)}-v_{k}^{(2)} \\
& v_{k}^{(12)}=v_{k}^{(1)}-v_{k}^{(2)}
\end{aligned}
$$

and 


$$
\begin{aligned}
& \varepsilon=\varepsilon^{(1)}+\varepsilon^{(2)} \\
& h=h^{(1)}+h^{(2)}
\end{aligned}
$$

The heat flux vector of the mixture is

$$
q_{k}=q_{k}^{(1)}-\varepsilon^{(1)}\left(v_{k}^{(1)}-v_{k}\right)+q_{k}^{(2)}-\varepsilon^{(2)}\left(v_{k}^{(2)}-v_{k}\right)
$$

The free energy density of the mixture is defined by

$$
\psi=\varepsilon-\theta \eta
$$

where $\theta$ is the absolute temperature of the constituents and the mixture, and $\eta$ is the entropy density of the mixture. Substitution of Equation (23) into (19) yields

$$
\dot{\psi}+\dot{\theta} \eta+\theta \dot{\eta}=q_{k, k}+h+t_{k l}^{(1)} a_{k l}^{(1)}+t_{k l}^{(2)} a_{k l}^{(2)}+m_{k l}^{(1)} b_{l k}^{(1)}+m_{k l}^{(2)} b_{l k}^{(2)}-\hat{p}_{k}^{(1)} v_{k}^{(12)}-\hat{m}_{k}^{(1)} v_{k}^{(12)}
$$

\subsection{Clausius-Duhem inequality}

The second law of thermodynamics is expressed as

$$
\dot{\eta}-\frac{1}{\theta} q_{k, k}+q_{k} \frac{\theta_{, k}}{\theta^{2}}-\frac{1}{\theta} h \geq 0
$$

Substitution of Equation (24) into (25) yields the Clausius-Duhem inequality

$$
-(\dot{\psi}+\dot{\theta} \eta)+t_{k l}^{(1)} a_{k l}^{(1)}+t_{k l}^{(2)} a_{k l}^{(2)}+m_{k l}^{(1)} b_{l k}^{(1)}+m_{k l}^{(2)} b_{l k}^{(2)}-\hat{p}_{k}^{(1)} v_{k}^{(12)}-\hat{m}_{k}^{(1)} v_{k}^{(12)}+q_{k} \frac{\theta_{, k}}{\theta} \geq 0
$$

\section{CONSTITUTIVE MODELING}

The parameters associated with field equations (11), (14), (17), (24) and the Clausius-Duhem inequality of Equation (26) are related by the constitutive equations. The constitutive independent and dependant variables are the sets $I$ and $D$, respectively, given by

$$
\begin{aligned}
& I=\left(\theta, \varepsilon_{k l}^{(1)}, \varepsilon_{k l}^{(2)}, \gamma_{k l}^{(1)}, \gamma_{k l}^{(2)}, v_{m}^{(12)}, v_{m}^{(12)}\right) \\
& D=\left(\psi, \eta, t_{k l}^{(1)}, t_{k l}^{(2)}, m_{k l}^{(1)}, m_{k l}^{(2)}, q_{m},-\hat{p}_{m}^{(1)},-\hat{m}_{m}^{(1)}\right)
\end{aligned}
$$

All the independent variables are frame-independent except $v_{m}^{(12)}$ and $v_{m}^{(12)}$. The admissibility for using these quantities has been established (Eringen, 2003). The parameters $\rho, \hat{p}_{m}^{(2)}$, and $\hat{m}_{m}^{(2)}$ are uniquely determined by Equations (11), (14), and (17), respectively, and thus are not included in 
sets $I$ and $D$. The free energy density is assumed to be a function of the static independent variables

$$
\psi=\tilde{\psi}\left(\theta, \varepsilon_{k l}^{(1)}, \varepsilon_{k l}^{(2)}, \gamma_{l k}^{(1)}, \gamma_{l k}^{(2)}\right)
$$

where the superposed $\sim$ denotes a response function. Using the chain rule, the material derivative of the free energy is given by

$$
\dot{\psi}=\frac{\partial \tilde{\psi}}{\partial \theta} \dot{\theta}+\frac{\partial \tilde{\psi}}{\partial \varepsilon_{k l}^{(1)}} a_{k l}^{(1)}+\frac{\partial \tilde{\psi}}{\partial \varepsilon_{k l}^{(2)}} a_{k l}^{(2)}+\frac{\partial \tilde{\psi}}{\partial \gamma_{l k}^{(1)}} b_{l k}^{(1)}+\frac{\partial \tilde{\psi}}{\partial \gamma_{l k}^{(2)}} b_{l k}^{(2)}
$$

Substituting Equation (29) into Equation (26) yields

$$
\begin{aligned}
-\left(\frac{\partial \tilde{\psi}}{\partial \theta}+\eta\right) \dot{\theta}+\left(t_{k l}^{(1)}-\frac{\partial \tilde{\psi}}{\partial \varepsilon_{k l}^{(1)}}\right) & a_{k l}^{(1)}+\left(t_{k l}^{(2)}-\frac{\partial \tilde{\psi}}{\partial \varepsilon_{k l}^{(2)}}\right) a_{k l}^{(2)}+\left(m_{k l}^{(1)}-\frac{\partial \tilde{\psi}}{\partial \gamma_{l k}^{(1)}}\right) b_{l k}^{(1)} \\
& +\left(m_{k l}^{(2)}-\frac{\partial \tilde{\psi}}{\partial \gamma_{l k}^{(2)}}\right) b_{l k}^{(2)}-\hat{p}_{k}^{(1)} v_{k}^{(12)}-\hat{m}_{k}^{(1)} v_{k}^{(12)}+q_{k} \frac{\theta_{k}}{\theta} \geq 0
\end{aligned}
$$

If Equation (30) is to be satisfied for arbitrary values of $\dot{\theta}, a_{k l}^{(n)}$, and $b_{l k}^{(n)}$ then

$$
\eta=-\frac{\partial \tilde{\psi}}{\partial \theta} \quad t_{k l}^{(1)}=\frac{\partial \tilde{\psi}}{\partial \varepsilon_{k l}^{(1)}} \quad t_{k l}^{(2)}=\frac{\partial \tilde{\psi}}{\partial \varepsilon_{k l}^{(2)}} \quad m_{k l}^{(1)}=\frac{\partial \tilde{\psi}}{\partial \gamma_{l k}^{(1)}} \quad m_{k l}^{(2)}=\frac{\partial \tilde{\psi}}{\partial \gamma_{l k}^{(2)}}
$$

Substituting Equation (31) back into Equation (30)

$$
-\hat{p}_{m}^{(1)} v_{m}^{(12)}-\hat{m}_{m}^{(1)} v_{m}^{(12)}+q_{m} \frac{\theta_{m}}{\theta} \geq 0
$$

The inequality of Equation (32) implies a set of thermodynamic forces $Y$ and fluxes $J$ (Eringen, 1999) given by

$$
\begin{aligned}
& Y=\left(v_{m}^{(12)}, v_{m}^{(12)}, \frac{\theta_{m}}{\theta}\right) \\
& J=\left(-\hat{p}_{m}^{(1)},-\hat{m}_{m}^{(1)}, q_{m}\right)
\end{aligned}
$$

Using a similar approach to that used by Eringen (Eringen, 2003) the constitutive equations associated with these quantities are 


$$
\begin{aligned}
& \hat{p}_{k}^{(1)}=-\frac{\partial \Phi}{\partial v_{k}^{(12)}} \\
& \hat{m}_{k}^{(1)}=-\frac{\partial \Phi}{\partial v_{k}^{(12)}} \\
& q_{k}=\frac{\partial \Phi}{\partial\left(\theta_{, k} / \theta\right)}
\end{aligned}
$$

where $\Phi$ is the dissipation potential (Eringen, 1999).

The specific free energy of the mixture is

$$
\begin{aligned}
\psi=S_{0}- & \eta_{0} T-\frac{C_{0}}{2 T_{0}} T^{2}-A_{k l}^{(1)} T \varepsilon_{k l}^{(1)}-A_{k l}^{(2)} T \varepsilon_{k l}^{(2)}-B_{k l}^{(1)} T \gamma_{k l}^{(1)}-B_{k l}^{(2)} T \gamma_{k l}^{(2)} \\
+ & \frac{1}{2}\left(A_{k l m n}^{(1)} \varepsilon_{k l}^{(1)} \varepsilon_{m n}^{(1)}+B_{k l m n}^{(1)} \gamma_{k l}^{(1)} \gamma_{m n}^{(1)}+2 C_{k l m n}^{(1)} \varepsilon_{k l}^{(1)} \gamma_{m n}^{(1)}\right) \\
+ & \frac{1}{2}\left(A_{k l m n}^{(2)} \varepsilon_{k l}^{(2)} \varepsilon_{m n}^{(2)}+B_{k l m n}^{(2)} \gamma_{k l}^{(2)} \gamma_{m n}^{(2)}+2 C_{k l m n}^{(2)} \varepsilon_{k l}^{(2)} \gamma_{m n}^{(2)}\right)
\end{aligned}
$$

where the subscript 0 denotes constants or variables in their natural state, that is, a state that is free of stress and couple stress. $S_{0}$ is the free energy in the natural state, $C_{0}$ is a constant relating temperature and free energy in the natural state, $T_{0}$ is the ambient temperature, $T$ is the change in ambient temperature resulting in the current temperature $\theta\left(\theta=T_{\mathrm{o}}+T\right.$, where $|T|$ is much less than $T_{0}, T_{0}>0$ ), and $A_{k l}^{(n)}, B_{k l}^{(n)}, A_{k l m n}^{(n)}, B_{k l m n}^{(n)}, C_{k l m n}^{(n)}$ are material moduli. The dissipation potential of the mixture is given by

$$
2 \Phi=\xi v_{k}^{(12)} v_{k}^{(12)}+2 \zeta v_{k}^{(12)} \frac{\theta_{, k}}{\theta}+K \frac{\theta_{, k} \theta_{, k}}{\theta^{2}}+\varpi v_{k}^{(12)} v_{k}^{(12)}
$$

where $\xi$ is the momentum generation coefficient due to velocity difference, $\zeta$ is the heat generation for unit change in velocity, $K$ is the classical Fourier constant, and $\varpi$ is the momentum generation due to difference in gyrations. The linear constitutive equations are obtained by substituting (35) and (36) into (31) and (34), 


$$
\begin{aligned}
& \eta=\eta_{0}+\frac{C_{0}}{T_{0}} T+A_{k l}^{(1)} \varepsilon_{k l}^{(1)}+B_{k l}^{(1)} \gamma_{k l}^{(1)}+A_{k l}^{(2)} \varepsilon_{k l}^{(2)}+B_{k l}^{(2)} \gamma_{k l}^{(2)} \\
& t_{k l}^{(n)}=-A_{k l}^{(n)} T+A_{k l m n}^{(n)} \varepsilon_{m n}^{(n)}+C_{k l m n}^{(n)} \gamma_{m n}^{(n)} \\
& m_{k l}^{(n)}=-B_{k l}^{(n)} T+B_{l k m n}^{(n)} \gamma_{m n}^{(n)}+C_{k l m n}^{(n)} \varepsilon_{m n}^{(n)} \\
& \hat{p}_{k}^{(1)}=-\xi v_{k}^{(12)}-\zeta \frac{T_{, k}}{T_{0}} \\
& \hat{m}_{k}^{(1)}=-\varpi v_{k}^{(12)} \\
& q_{k}=\zeta v_{k}^{(12)}+K \frac{T_{, k}}{T_{0}}
\end{aligned}
$$

For isotropic constituents (Eringen, 1999)

$$
\begin{aligned}
& B_{k l}^{(n)}=0_{k l} \\
& C_{k l m n}^{(n)}=0_{k l m n} \\
& A_{k l}^{(n)}=\beta_{0}^{(n)} \delta_{k l} \\
& A_{k l m n}^{(n)}=\lambda^{(n)} \delta_{k l} \delta_{m n}+\left[\mu^{(n)}+\kappa^{(n)}\right] \delta_{k m} \delta_{l n}+\mu^{(n)} \delta_{k n} \delta_{l m} \\
& B_{k l m n}^{(n)}=\alpha^{(n)} \delta_{k l} \delta_{m n}+\beta^{(n)} \delta_{k n} \delta_{l m}+\gamma^{(n)} \delta_{k m} \delta_{l n}
\end{aligned}
$$

where $0_{k l}$ and $0_{k l m n}$ are the components of the second- and fourth-order null tensors, respectively; $\alpha^{(n)}, \beta^{(n)}, \gamma^{(n)}, \lambda^{(n)}, \mu^{(n)}, \kappa^{(n)}$ are the six micropolar elastic constants; and $\beta_{0}^{(n)}$ is a thermal expansion constant for determining micropolar isotropic behavior. Substitution of Equation (38) into Equation (37) yields

$$
\begin{gathered}
\eta=\eta_{0}+\frac{C_{0}}{T_{0}} T+\beta_{0}^{(1)} \varepsilon_{k k}^{(1)}+\beta_{0}^{(2)} \varepsilon_{k k}^{(2)} \\
t_{k l}^{(n)}=\left[-\beta_{0}^{(n)} T+\lambda^{(n)} \varepsilon_{m m}^{(n)}\right] \delta_{k l}+\left[\mu^{(n)}+\kappa^{(n)}\right] \varepsilon_{k l}^{(n)}+\mu^{(n)} \varepsilon_{l k}^{(n)} \\
m_{k l}^{(n)}=\alpha^{(n)} \delta_{k l} \gamma_{m m}^{(n)}+\beta^{(n)} \gamma_{l k}^{(n)}+\gamma^{(n)} \gamma_{k l}^{(n)} \\
\hat{p}_{k}^{(1)}=-\xi v_{k}^{(12)}-\zeta \frac{T_{, k}}{T_{0}} \\
\hat{m}_{k}^{(1)}=-\varpi v_{k}^{(12)} \\
q_{k}=\zeta v_{k}^{(12)}+K \frac{T_{, k}}{T_{0}}
\end{gathered}
$$

Equation (39) - (44) describes the constitutive response of the constituents, the entropy density of the mixture, the interaction parameters, and the mixture heat flux vector. The overall behavior of the constituents is governed by the constitutive response as well as the balance equations 
discussed in Section 4. Substitution of Equations (39) - (44) into Equations (12), (15), and (24) yield, respectively,

$$
\begin{gathered}
\rho^{(1)} \ddot{x}_{l}^{(1)}-\lambda^{(1)} \varepsilon_{m m, l}^{(1)}-\left[\mu^{(1)}+\kappa^{(1)}\right] \varepsilon_{k l, k}^{(1)}-\mu^{(1)} \varepsilon_{l k, k}^{(1)}+\xi v_{l}^{(12)}=0 \\
\rho^{(2)} \ddot{x}_{l}^{(2)}-\lambda^{(2)} \varepsilon_{m m, l}^{(2)}-\left[\mu^{(2)}+\kappa^{(2)}\right] \varepsilon_{k l, k}^{(2)}-\mu^{(2)} \varepsilon_{l k, k}^{(2)}-\xi v_{l}^{(12)}=0 \\
\rho^{(1)} j^{(1)} \dot{v}_{l}^{(1)}-\alpha^{(1)} \gamma_{m m, l}^{(1)}-\beta^{(1)} \gamma_{k l, k}^{(1)}-\gamma^{(1)} \gamma_{l k, k}^{(1)}-\varepsilon_{l m n}\left[\left(\mu^{(1)}+\kappa^{(1)}\right) \varepsilon_{m n}^{(1)}+\mu^{(1)} \varepsilon_{n m}^{(1)}\right]+\varpi v_{l}^{(12)}=0 \\
\rho^{(2)} j^{(2)} \dot{v}_{l}^{(2)}-\alpha^{(2)} \gamma_{m m, l}^{(2)}-\beta^{(2)} \gamma_{k l, k}^{(2)}-\gamma^{(2)} \gamma_{l k, k}^{(2)}-\varepsilon_{l m n}\left[\left(\mu^{(2)}+\kappa^{(2)}\right) \varepsilon_{m n}^{(2)}+\mu^{(2)} \varepsilon_{n m}^{(2)}\right]-\varpi v_{l}^{(12)}=0 \\
\zeta v_{k, k}^{(12)}-\hat{p}_{k}^{(1)} v_{k}^{(12)}-\hat{m}_{k}^{(1)} v_{k}^{(12)}-T \beta_{0}^{(1)} a_{k k}^{(1)}-T \beta_{0}^{(2)} a_{k k}^{(2)}=0
\end{gathered}
$$

where isothermal conditions are assumed and body force densities, body couple densities, heat source densities, and temperature gradients have been neglected.

The theory developed in this section models the interaction of the two grids as a dissipative mechanism, which is consistent with the original framework of the theory presented previously for a mixture of a solid and fluid (Eringen, 2003). However, the interaction of the two grids does not necessarily need to be modeled as a dissipative process as this interaction may potentially be purely elastic in nature. For such a case, grid interaction terms could be added to the strain energy function of Equation (35) instead of the dissipation function of Equation (36). In this manner, a non-dissipative approach can be used to model the interaction of the grids.

\section{EXAMPLE: TWO-DIMENSIONAL CELLULAR SOLIDS}

The constitutive framework developed in Section 5 is for a general mixture of two micropolar elastic solids. To demonstrate the application of the proposed theory, the constitutive response of a two-dimensional cellular material with a distribution of cell sizes is determined. Although the typical honeycomb structure would be an excellent candidate for the application of this theory, honeycomb lattices usually have a very narrow distribution of cell sizes. Structures with a significant distribution of cell sizes are found in many varieties of wood. For example, the microstructure of a cross-sectional slice of balsa wood is shown in Figure 4. To determine the constitutive response of this two-dimensional natural cellular material, its cell size distribution is matched with the cell size distribution of the conceptual combined triangular grid (Figure2). The triangular grid was selected for this research because of its simplicity and because of the availability of a micropolar solution in the literature (Ostoja-Starzewski, 2002). Alternatively, a hexagonal lattice could be used that may more accurately represent the geometry of the balsa wood cells; however, the micropolar solution to a hexagonal lattice must be established first. The conceptual triangular grid, which then represents the microstructure in Figure 4, is homogenized in two steps, namely, the micropolar homogenization step in which each individual grid is converted to an effective micropolar continuum and the micropolar mixture theory homogenization step where the individual micropolar continua are superimposed using the micropolar mixture theory. This yields the equivalent continuum whose mechanical behavior represents the mechanical behavior of the natural two-dimensional cellular material. It is important to note that since the micropolar mixture theory combines two equivalent micropolar 
continua and not the individual lattices, the model does not directly model the conceptual combined triangular grid shown at the top of Figure 2. In the current section, the micropolar homogenization of a single triangular grid is discussed followed by the details of the micropolar mixture theory homogenization and the resulting constitutive response of the mixture.

\subsection{Triangular Lattice Homogenization}

The equivalent micropolar continuum for a single triangular grid composed of Timoshenko beams has been previously established (Ostoja-Starzewski, 2002). In this formulation, given the length of the strut of the $n^{\text {th }}$ constituent $l^{(n)}$ (Figure 5), cross-sectional width and height of the strut of the $n^{\text {th }}$ constituent $s^{(n)}$, the Young's modulus of the material composing the struts of the $n^{\text {th }}$ constituent $E_{\text {strut }}^{(n)}$, and the shear modulus of the material composing the struts of the $n^{\text {th }}$ constituent $G_{\text {strut }}^{(n)}$, the equivalent in-plane isotropic micropolar elastic moduli for a twodimensional equivalent continuum of thickness $s^{(n)}$ are

$$
\begin{aligned}
\lambda^{(n)}=\mu^{(n)} & =\frac{3}{8} \frac{Q^{(n)}-R^{(n)}}{s^{(n)}} \\
\kappa^{(n)} & =\frac{3}{2} \frac{R^{(n)}}{s^{(n)}} \\
\gamma^{(n)} & =\frac{3}{2} \frac{S^{(n)}}{s^{(n)}} \\
\alpha^{(n)} & =\beta^{(n)}=0
\end{aligned}
$$

where

$$
\begin{gathered}
Q^{(n)}=\frac{2 E_{\text {strut }}^{(n)} A^{(n)}}{l^{(n)} \sqrt{3}} \\
R^{(n)}=\frac{24 E_{\text {strut }}^{(n)} I^{(n)}}{\left[l^{(n)}\right]^{3} \sqrt{3}} \frac{1}{1+T^{(n)}} \\
S^{(n)}=\frac{2 E_{\text {strut }}^{(n)} I^{(n)}}{l^{(n)} \sqrt{3}} \\
\left.T^{(n)}=\frac{E_{\text {strut }}^{(n)}\left(\frac{S^{(n)}}{G_{\text {strut }}^{(n)}}\right)^{2}}{l^{(n)}}\right)^{2}
\end{gathered}
$$

In Equation (49) $A^{(n)}$ and $I^{(n)}$ are the cross-sectional area and moment of inertia of the struts of the $n^{\text {th }}$ constituent, respectively, given by 


$$
A^{(n)}=\left[s^{(n)}\right]^{2} \quad I^{(n)}=\frac{\left[s^{(n)}\right]^{4}}{12}
$$

It is important to note that Equation (48) differs from the analogous equations of OstojaStarzewski (Ostoja-Starzewski, 2002) by the strut thickness in the denominator. The inclusion of the strut thickness serves to normalize Equation (48) with respect to an arbitrary thickness, similar to the classical plate theory (Christensen, 2005). These equations assume a plane state of stress in the plane of the triangular lattice, as shown in Figure 5 . The relative density of the triangular lattice is (Gibson and Ashby, 1999)

$$
\rho_{r e l}^{(n)}=2 \sqrt{3} \frac{s^{(n)}}{l^{(n)}}\left[1-\frac{\sqrt{3}}{2} \frac{s^{(n)}}{l^{(n)}}\right]
$$

It is noted that it has been shown (Ostoja-Starzewski, 2002) that for relative densities of $80 \%$ and higher, the strut width becomes too large for Equation (48) to accurately predict the elastic properties of the equivalent micropolar continuum.

Consider the case of the equivalent micropolar continuum loaded in uniaxial tension parallel to the $\mathbf{e}_{1}$ basis vector shown in Figure 5, in which the strains are

$$
\varepsilon_{11}^{(n)} \quad \varepsilon_{22}^{(n)}=\varepsilon_{33}^{(n)} \quad \varepsilon_{23}^{(n)}=\varepsilon_{13}^{(n)}=\varepsilon_{12}^{(n)}=0
$$

where $\varepsilon_{11}^{(n)}$ is the applied uniaxial strain. The transverse stresses are

$$
t_{22}^{(n)}=t_{33}^{(n)}=0
$$

The in-plane Young's modulus $E^{(n)}$ and the Poisson's ratio $v^{(n)}$ of the $n^{\text {th }}$ constituent are defined as, respectively,

$$
\begin{gathered}
E^{(n)} \equiv \frac{t_{11}^{(n)}}{\varepsilon_{11}^{(n)}} \\
v^{(n)} \equiv-\frac{\varepsilon_{22}^{(n)}}{\varepsilon_{11}^{(n)}}
\end{gathered}
$$

Isothermal conditions are assumed. Substitution of Equation (52) into the $t_{11}^{(n)}$ component of Equation (40) yields

$$
t_{11}^{(n)}=\left[2 \lambda^{(n)} \frac{\varepsilon_{22}^{(n)}}{\varepsilon_{11}^{(n)}}+2 \mu^{(n)}+\kappa^{(n)}\right] \varepsilon_{11}^{(n)}
$$


Substituting Equations (52) and (53) into the $t_{22}^{(n)}$ component of Equation (40)

$$
t_{22}^{(n)}=0=\lambda^{(n)} \varepsilon_{11}^{(n)}+\left[2 \mu^{(n)}+2 \lambda^{(n)}+\kappa^{(n)}\right] \varepsilon_{22}^{(n)}
$$

Substitution of Equations (56) and (57) into (54) establishes the Young's modulus of the equivalent continuum of the $n^{\text {th }}$ constituent

$$
E^{(n)}=\frac{\left(2 \mu^{(n)}+\kappa^{(n)}\right)\left(3 \lambda^{(n)}+2 \mu^{(n)}+\kappa^{(n)}\right)}{2 \lambda^{(n)}+2 \mu^{(n)}+\kappa^{(n)}}
$$

Substitution of Equation (57) into (55) provides

$$
v^{(n)}=\frac{\lambda^{(n)}}{2 \mu^{(n)}+2 \lambda^{(n)}+\kappa^{(n)}}
$$

Now consider the case of pure shear in the $\mathbf{e}_{1}-\mathbf{e}_{2}$ plane of the equivalent micropolar continuum. Again, isothermal conditions are assumed to exist. The corresponding strain field is

$$
\varepsilon_{12}^{(n)}=\varepsilon_{21}^{(n)} \quad \varepsilon_{11}^{(n)}=\varepsilon_{22}^{(n)}=\varepsilon_{33}^{(n)}=\varepsilon_{13}^{(n)}=\varepsilon_{23}^{(n)}=0
$$

where $\gamma$ is the engineering shear strain. The in-plane shear modulus of the equivalent continuum of the $n^{\text {th }}$ constituent is defined as

$$
G^{(n)} \equiv \frac{t_{12}^{(n)}}{2 \varepsilon_{12}^{(n)}}
$$

Substitution of Equation (60) into (40) for the 1-2 component of stress

$$
G^{(n)}=\mu^{(n)}+\frac{\kappa^{(n)}}{2}
$$

It is noted that Equations (58), (59), and (62) are consistent with those reported elsewhere (Nowacki, 1974; Gauthier and Jahsman, 1975; Gauthier and Jahsman, 1976; Eringen, 1999)

\subsection{Micropolar Mixture of Triangular Lattices}

Although the constitutive and field equations govern the response of the micropolar mixture, the nature of the interactions, as represented by $\hat{p}_{k}^{(1)}$ and $\hat{m}_{k}^{(1)}$ have yet to be determined. For simplicity, it is assumed here that 


$$
\begin{aligned}
& u_{k}=u_{k}^{(1)}=u_{k}^{(2)} \\
& \phi_{k}=\phi_{k}^{(1)}=\phi_{k}^{(2)}
\end{aligned}
$$

Therefore

$$
\begin{array}{ll}
\varepsilon_{k l}=\varepsilon_{k l}^{(1)}=\varepsilon_{k l}^{(2)} & a_{k l}=a_{k l}^{(1)}=a_{k l}^{(2)} \\
\gamma_{k l}=\gamma_{k l}^{(1)}=\gamma_{k l}^{(2)} & b_{k l}=b_{k l}^{(1)}=b_{k l}^{(2)}
\end{array}
$$

where $u_{\mathrm{k}}, \phi_{\mathrm{k}}, \varepsilon_{\mathrm{kl}}, \gamma_{\mathrm{kl}}, a_{\mathrm{kl}}$ and $b_{\mathrm{kl}}$ are the kinematic quantities associated with the mixture. Hence by virtue of Equation (63) and in the absence of temperature gradients

$$
v_{k}^{(12)}=\hat{p}_{k}^{(1)}=0_{k} \quad v_{k}^{(12)}=\hat{m}_{k}^{(1)}=0_{k}
$$

The elastic mixture theory assumes that the stress $t_{\mathrm{ij}}$ and couple stress $m_{\mathrm{ij}}$ of the mixture are given by

$$
\begin{gathered}
t_{i j}=f^{(1)} t_{i j}^{(1)}+f^{(2)} t_{i j}^{(2)} \\
m_{i j}=f^{(1)} m_{i j}^{(1)}+f^{(2)} m_{i j}^{(2)}
\end{gathered}
$$

where $f^{(1)}$ and $f^{(2)}$ are the volume fractions of constituents 1 and 2, respectively, in the mixture. For the binary mixture considered in this study $f^{(1)}+f^{(2)}=1$.

The assumptions of Equations (63) and (66) are the simplest assumptions for the interaction of the constituents. In fact, Equations (63) imply that no internal interactions exist between the micropolar constituents. A possible physical interpretation of this assumption with regards to the conceptual combined triangular grid shown in Figure 2 is that there are no locations in which the two grids are bonded together. If on the other hand, the grids are "welded" at their junction points, then the assumption of Equation (63) must be modified appropriately.

Consider again the uniaxial deformation described by Equations (52) and (53). If the same deformation field (here $\varepsilon_{\mathrm{ij}}$ are the components of strain of the mixture) is applied to the binary mixture, the Young's modulus $E$ and Poisson's ratio $v$ of the mixture are, respectively,

$$
\begin{gathered}
E \equiv \frac{t_{11}}{\varepsilon_{11}} \\
v \equiv-\frac{\varepsilon_{22}}{\varepsilon_{11}}
\end{gathered}
$$

Substitution of Equations (58) and (66) ${ }_{1}$ into (67) reveals 


$$
\begin{aligned}
E & =f^{(1)} E^{(1)}+f^{(2)} E^{(2)} \\
& =f^{(1)} \frac{\left(2 \mu^{(1)}+\kappa^{(1)}\right)\left(3 \lambda^{(1)}+2 \mu^{(1)}+\kappa^{(1)}\right)}{2 \lambda^{(1)}+2 \mu^{(1)}+\kappa^{(1)}}+f^{(2)} \frac{\left(2 \mu^{(2)}+\kappa^{(2)}\right)\left(3 \lambda^{(2)}+2 \mu^{(2)}+\kappa^{(2)}\right)}{2 \lambda^{(2)}+2 \mu^{(2)}+\kappa^{(2)}}
\end{aligned}
$$

Since the normal strains in the constituents are equal to those in the mixture, Equation (59) is equal to Equation (68)

$$
\begin{aligned}
v & =v^{(1)}=v^{(2)} \\
& =\frac{\lambda^{(1)}}{2 \mu^{(1)}+2 \lambda^{(1)}+\kappa^{(1)}}=\frac{\lambda^{(2)}}{2 \mu^{(2)}+2 \lambda^{(2)}+\kappa^{(2)}}
\end{aligned}
$$

When the strain field described by Equation (60) is applied to the mixture for the case of pure shear, the shear modulus of the mixture $G$ is

$$
G \equiv \frac{t_{12}}{2 \varepsilon_{12}}
$$

Substitution of Equations (61), (62), and (66) into (71)

$$
G=f^{(1)} G^{(1)}+f^{(2)} G^{(2)}=f^{(1)}\left[\mu^{(1)}+\frac{\kappa^{(1)}}{2}\right]+f^{(2)}\left[\mu^{(2)}+\frac{\kappa^{(2)}}{2}\right]
$$

In a similar manner, the micropolar moduli of the mixture can be determined.

\subsection{Application of Model to Balsa Wood}

A cross sectional slice of balsa wood closely approximates a two-dimensional cellular structure with a distribution of cell sizes. Figure 4, which is an image of an axial cross-section of balsa wood, shows three types of cells. Most of the volume is occupied by nearly hexagonal normal cells, with parallel bands of rectangular ray cells. The larger sap cells occupy a much smaller volume than the normal and ray cells over the entire cross section (Figure 4 is focused on an area crowded with sap cells), thus their relative volume fraction is insignificant compared to those of the normal and ray cells.

A binary mixture model was constructed in which the $1^{\text {st }}$ and $2^{\text {nd }}$ constituents were the equivalent continua of the normal and ray cells, respectively. The structural and mechanical parameters for the two lattices are shown in Table 1 . The values of $s^{(n)}, E^{(n)}$, and $f^{(n)}$ were previously determined by Easterling et al. (Easterling et al., 1982). The values of $l^{(n)}$ were determined by equating the average cell areas reported by Easterling et al. (Easterling et al., 1982) with triangular cell areas for the triangular lattice. The values of $G^{(n)}$ were calculated assuming a cell wall Poisson's ratio of 0.33 . 
Using Equations (48) - (50), (58), (59), (62), (69), (70), and (72), the in-plane Young's modulus, Poisson's ratio, and shear modulus of balsa wood were predicted to be $376 \mathrm{MPa}, 0.25$, and 150 $\mathrm{MPa}$, respectively. It is important to note that, as stated earlier, these equations assume a plane state of stress, and a specimen of balsa wood tested experimentally is likely to have struts that experience plane strain as the strut thicknesses are much large than strut widths. However, the effect of the plane stress assumption on the final predicted values of the mechanical properties is likely quite small (Ostoja-Starzewski, 2002). Experimental measurements of in-plane Young's modulus of balsa wood range from $10-300 \mathrm{MPa}$ (Easterling et al., 1982) . Therefore, the predicted Young's modulus is in rough agreement with the measured value. The model did not take into consideration the presence of sap channels and imperfections in the material, which may explain the overestimate of the predicted properties.

\section{SUMMARY}

An analytical modeling approach has been developed to predict the elastic properties of cellular materials without the need for complex and inefficient FEA modeling. The modeling approach directly accounts for the distribution of cell geometries that are present in most cellular materials, and provides for the opportunity for efficient analysis, optimization, and design of cellular materials. The approach combines mixture theory and micropolar elasticity theory to predict elastic response of cellular materials to a wide range of loading conditions. It is important to note that despite the inefficiency of FEA modeling, it has the potential to be more accurate than analytical modeling.

The modeling approach was applied to the two-dimensional balsa wood material. Predicted properties were in good agreement with experimentally-determined properties. This agreement demonstrates that the model has the potential to predict the elastic response of other cellular solids, such as open cell and closed cell foams.

\section{ACKNOWLEDGMENTS}

This research was jointly sponsored by the Michigan Tech Research Excellence fund, the Department of Mechanical Engineering - Engineering Mechanics at Michigan Tech, and NASA grant \#NNL04AA85G. The Authors are also grateful to Mr. Timothy Walter for his assistance with Figure 1, and to Dr. Dennis Kunkel for his permission to use the image of balsa wood in Figure 4. 


\section{REFERENCES}

Bedford, A.,Stern, M., 1972. A Multi-Continuum Theory for Composite Elastic Materials. Acta Mechanica 14 (2-3) 85-102.

Christensen, R. M., 2005. Mechanics of Composite Materials. Dover Publications

Easterling, K. E., Harrysson, R., Gibson, L. J.,Ashby, M. F., 1982. On the Mechanics of Balsa and Other Woods. Proceedings of the Royal Society of London Series A-Mathematical Physical and Engineering Sciences 383 (1784) 31-41.

Eringen, A. C., 1999. Microcontinuum Field Theories 1: Foundations and Solids. SpringerVerlag New York, Inc.

Eringen, A. C., 2003. Micropolar mixture theory of porous media. Journal of Applied Physics 94 (6) 4184-4190.

Gauthier, R. D.,Jahsman, W. E., 1976. Bending of a Curved Bar of Micropolar Elastic Material. Journal of Applied Mechanics-Transactions of the ASME 43 Ser E (3) 502-503.

Gauthier, R. D.,Jahsman, W. E., 1975. A Quest for Micropolar Elastic Constants. Journal of Applied Mechanics-Transactions of the ASME 42 (2) 369-374.

Gibson, L. J.,Ashby, M. F., 1999. Cellular Solids: Structure and Properties. Cambridge University Press.

Hegemier, G. A., Gurtman, G. A.,Nayfeh, A. H., 1973. A Continuum Mixture Theory of Wave Propagation in Laminated and Fiber Reinforced Composites. International Journal of Solids and Structures 9 (3) 395-414.

Kanaun, S.,Tkachenko, O., 2006. Mechanical properties of open cell foams: Simulations by Laguerre tesselation procedure. International Journal of Fracture 140 (1-4) 305-312.

Li, K., Gao, X. L.,Subhash, G., 2006. Effects of Cell Shape and Strut Cross-Sectional Area Variations on the Elastic Properties of Three-Dimensional Open-Cell Foams. Journal of the Mechanics and Physics of Solids 54 (4) 783-806.

McNiven, H. D.,Mengi, Y., 1979a. Mathematical Model for the Linear Dynamic Behavior of Two Phase Periodic Materials. International Journal of Solids and Structures 15 (4) 271280.

McNiven, H. D.,Mengi, Y., 1979b. Mixture Theory for Elastic Laminated Composites. International Journal of Solids and Structures 15 (4) 281-302.

McNiven, H. D.,Mengi, Y., 1979c. Propagation of Transient Waves in Elastic Laminated Composites. International Journal of Solids and Structures 15 (4) 303-318.

Nayfeh, A. H.,Gurtman, G. A., 1974. A Continuum Approach to the Propagation of Shear Waves in Laminated Wave Guides. Journal of Applied Mechanics - Transactions of the ASME 41 (1) 106-119.

Noor, A. K.,Nemeth, M. P., 1980a. Analysis of Spatial Beam Like Lattices with Rigid Joints. Computer Methods in Applied Mechanics and Engineering 24 (1) 35-59.

Noor, A. K.,Nemeth, M. P., 1980b. Micropolar Beam Models for Lattice Grids with Rigid Joints. Computer Methods in Applied Mechanics and Engineering 21 (2) 249-263.

Nowacki, W., 1974. Theory of Micropolar Elasticity. Springer.

Ostoja-Starzewski, M., 2002. Lattice models in micromechanics. Applied Mechanics Reviews 55 (1) 35-59.

Roberts, A. P.,Garboczi, E. J., 2002. Elastic Properties of Model Random Three-Dimensional Open-Cell Solids. Journal of the Mechanics and Physics of Solids 50 (1) 33-55. 
Stern, M.,Bedford, A., 1972. Wave Propagation in Elastic Laminates Using a Multi-Continuum Theory. Acta Mechanica 15 (1-2) 21-38.

Sun, C. T.,Yang, T. Y., 1973. Continuum Approach Toward Dynamics of Gridworks. Journal of Applied Mechanics-Transactions of the ASME 40 (1) 186-192.

Truesdell, C.,Toupin, R., 1960. Encyclopedia of Physics. Springer-Verlag OHG.

Yoo, A.,Jasiuk, I., 2006. Couple-stress moduli of a trabecular bone idealized as a 3D periodic cellular network. Journal of Biomechanics 39 (12) 2241-2252.

Zhu, H. X., Hobdell, J. R.,Windle, A. H., 2000. Effects of cell irregularity on the elastic properties of open-cell foams. Acta Materialia 48 (20) 4893-4900. 


\section{TABLE CAPTIONS}

Table 1 - Properties of balsa wood lattices

\section{FIGURE CAPTIONS}

Figure 1 - Open cell polyurethane

Figure 2 - Modeling approach for cellular materials

Figure 3 - Motion of the mixture of two-constituent continua

Figure 4 - SEM image of a longitudinal cross-section of balsa wood (Image copyright Dennis Kunkel Microscopy, Inc., used with permission)

Figure 5 - Triangular lattice strut dimensions 
Table 1 - Properties of the equivalent lattices of balsa wood

\begin{tabular}{ccc} 
& Normal cells & Ray cells \\
\cline { 2 - 3 }$n$ & 1 & 2 \\
$s^{(n)}$ & $1.5 \mu \mathrm{m}$ & $1.5 \mu \mathrm{m}$ \\
$l^{(n)}$ & $44 \mu \mathrm{m}$ & $29 \mu \mathrm{m}$ \\
$f^{(n)}$ & $86 \%$ & $14 \%$ \\
$E_{\text {strut }}^{(n)}$ & $10 \mathrm{GPa}$ & $10 \mathrm{GPa}$ \\
$G_{\text {strut }}^{(n)}$ & $3.8 \mathrm{GPa}$ & $3.8 \mathrm{GPa}$
\end{tabular}




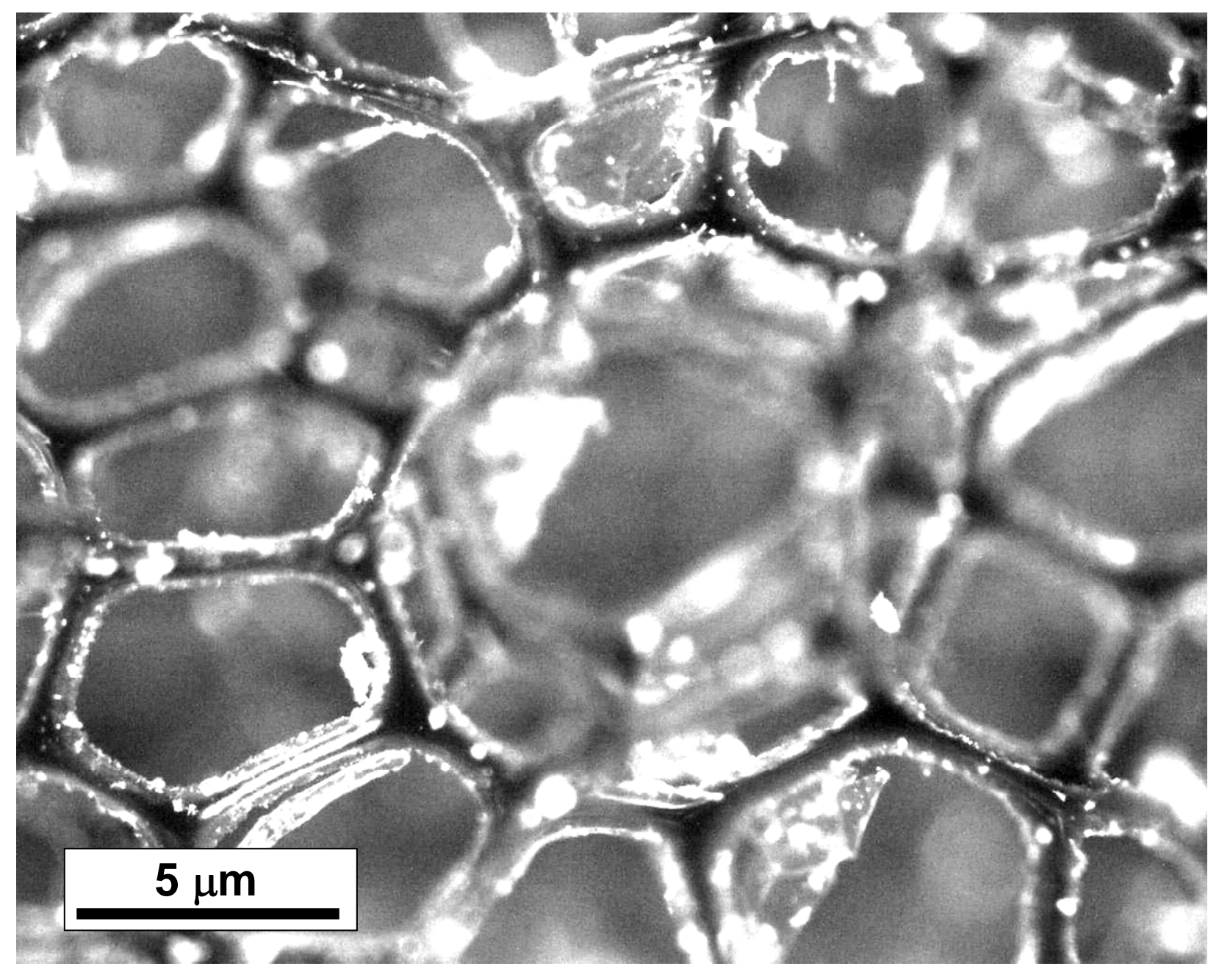

Figure 1 - Open cell polyurethane 


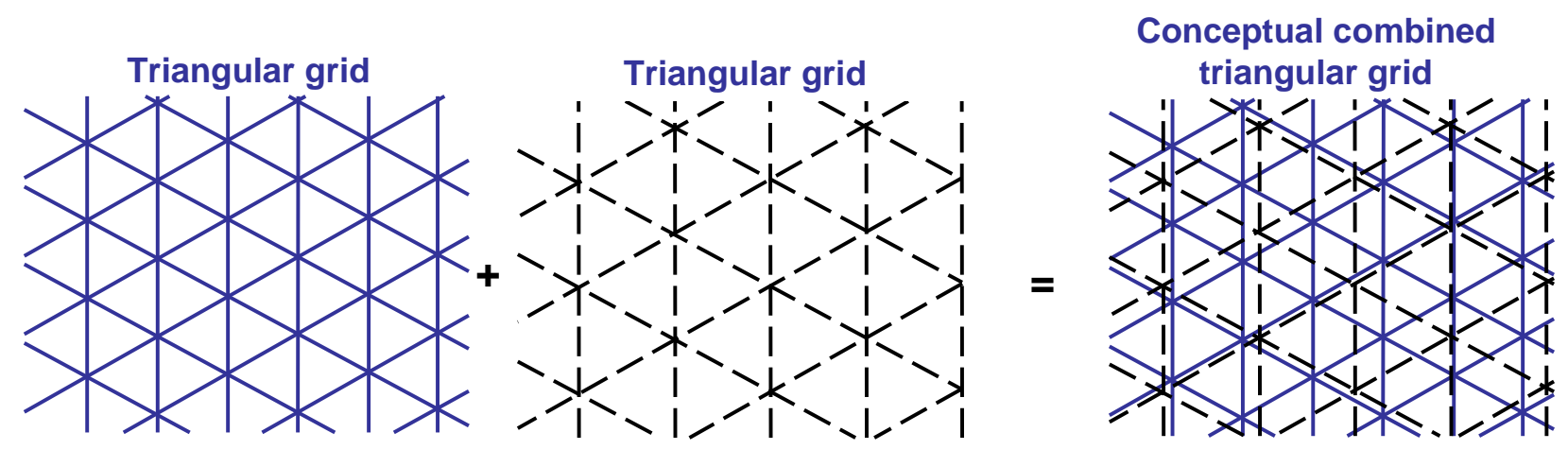

Micropolar

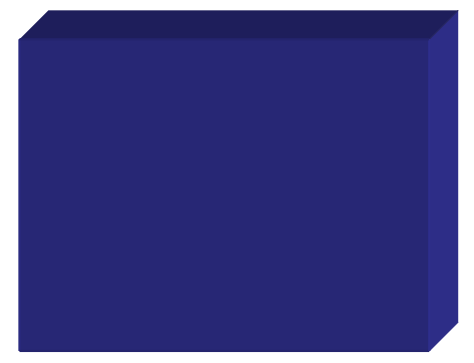

Micropolar constituent

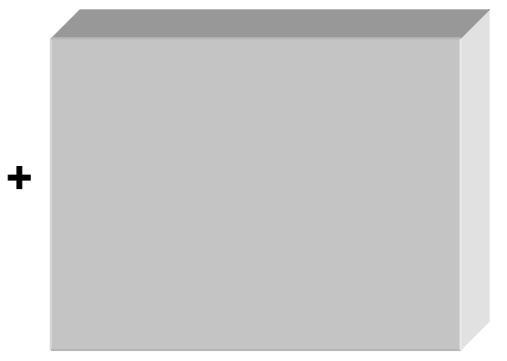

Micropolar constituent

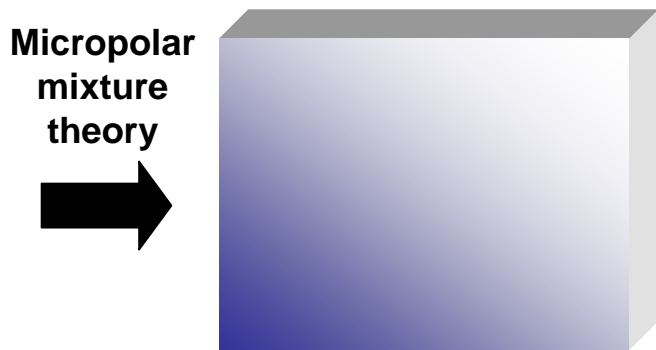

Micropolar mixture

Figure 2 - Modeling approach for cellular materials 


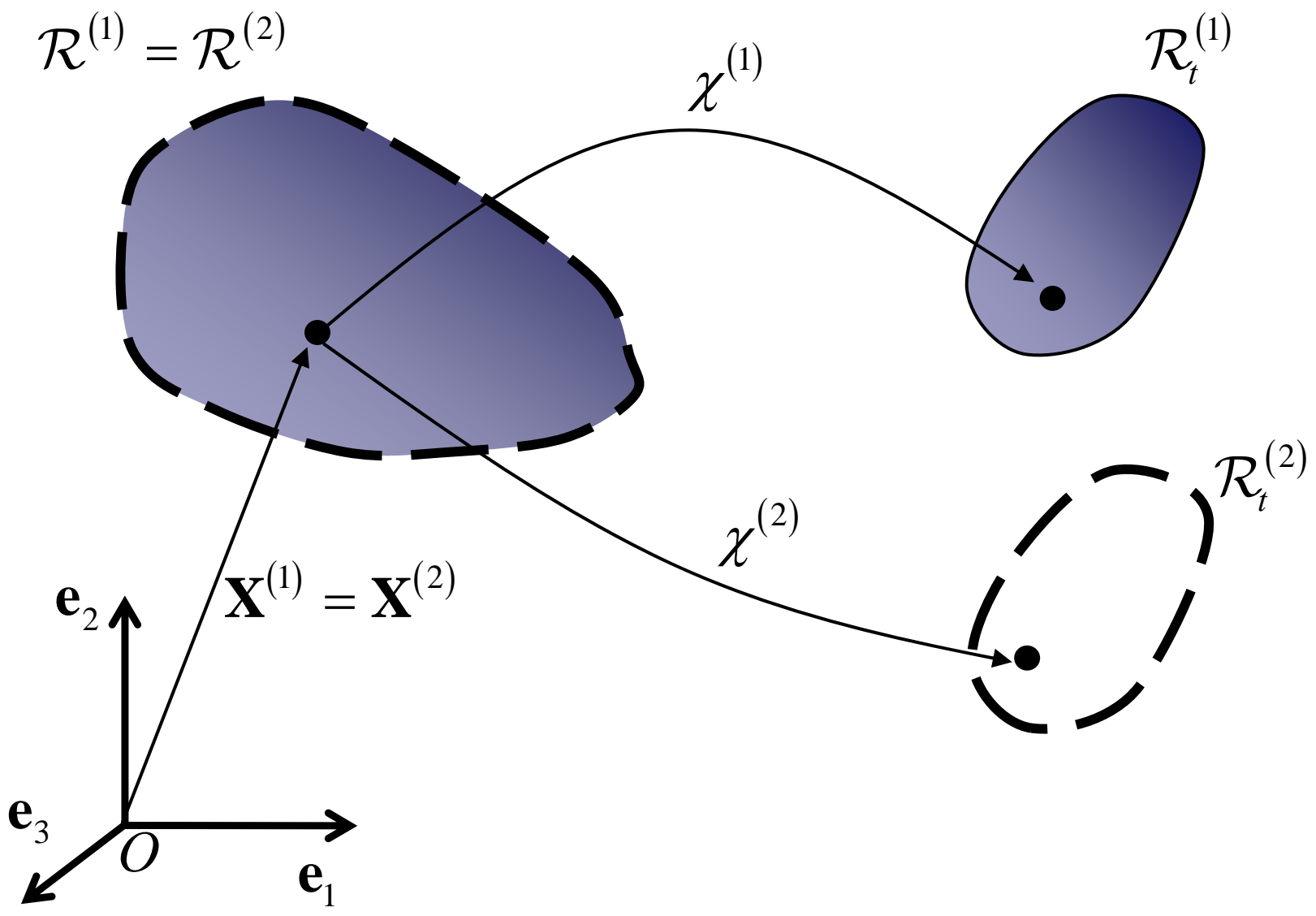

Figure 3 - Motion of the mixture of two-constituent continua 


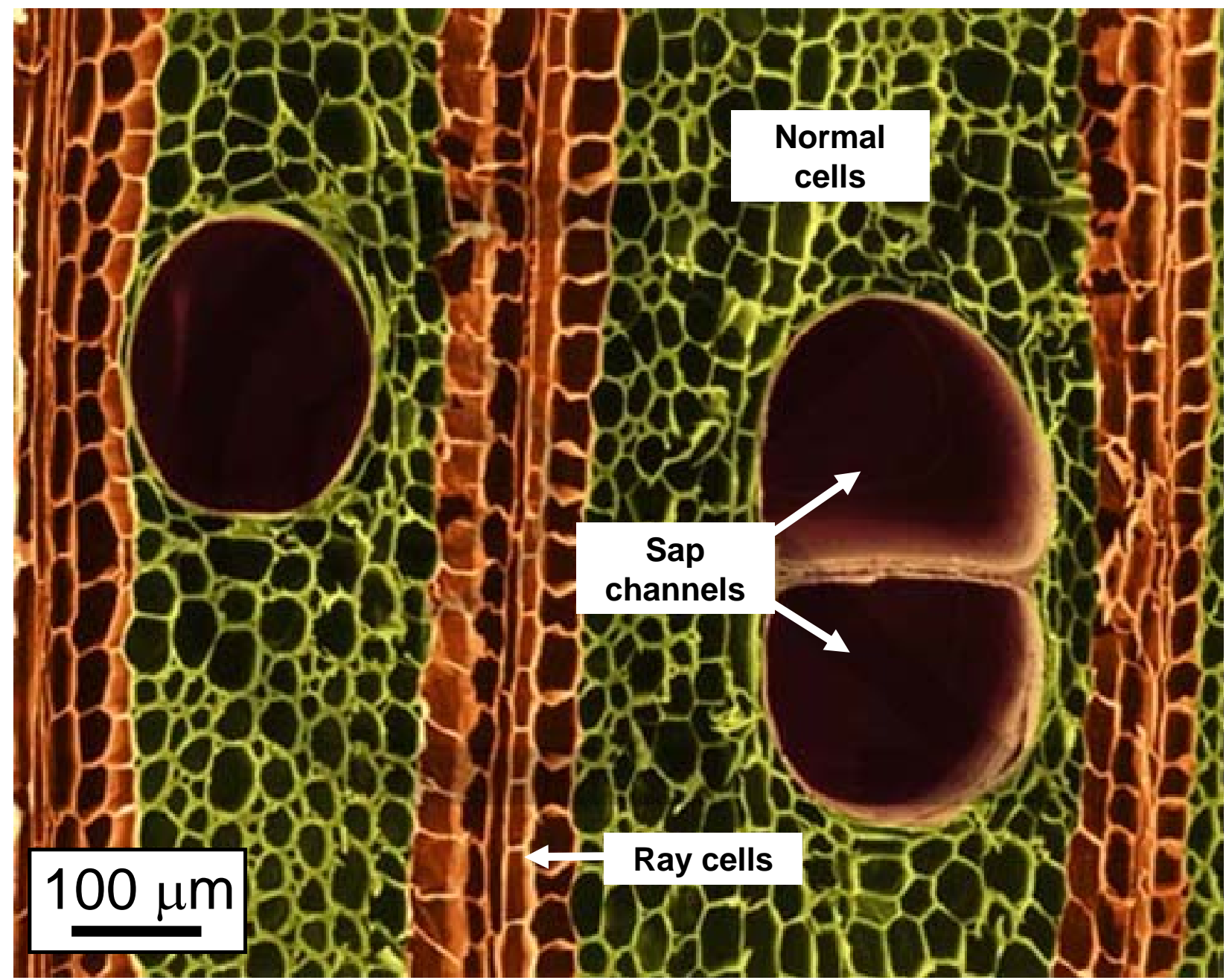

Figure 4 - SEM image of a longitudinal cross-section of balsa wood (Image copyright Dennis Kunkel Microscopy, Inc., used with permission) 


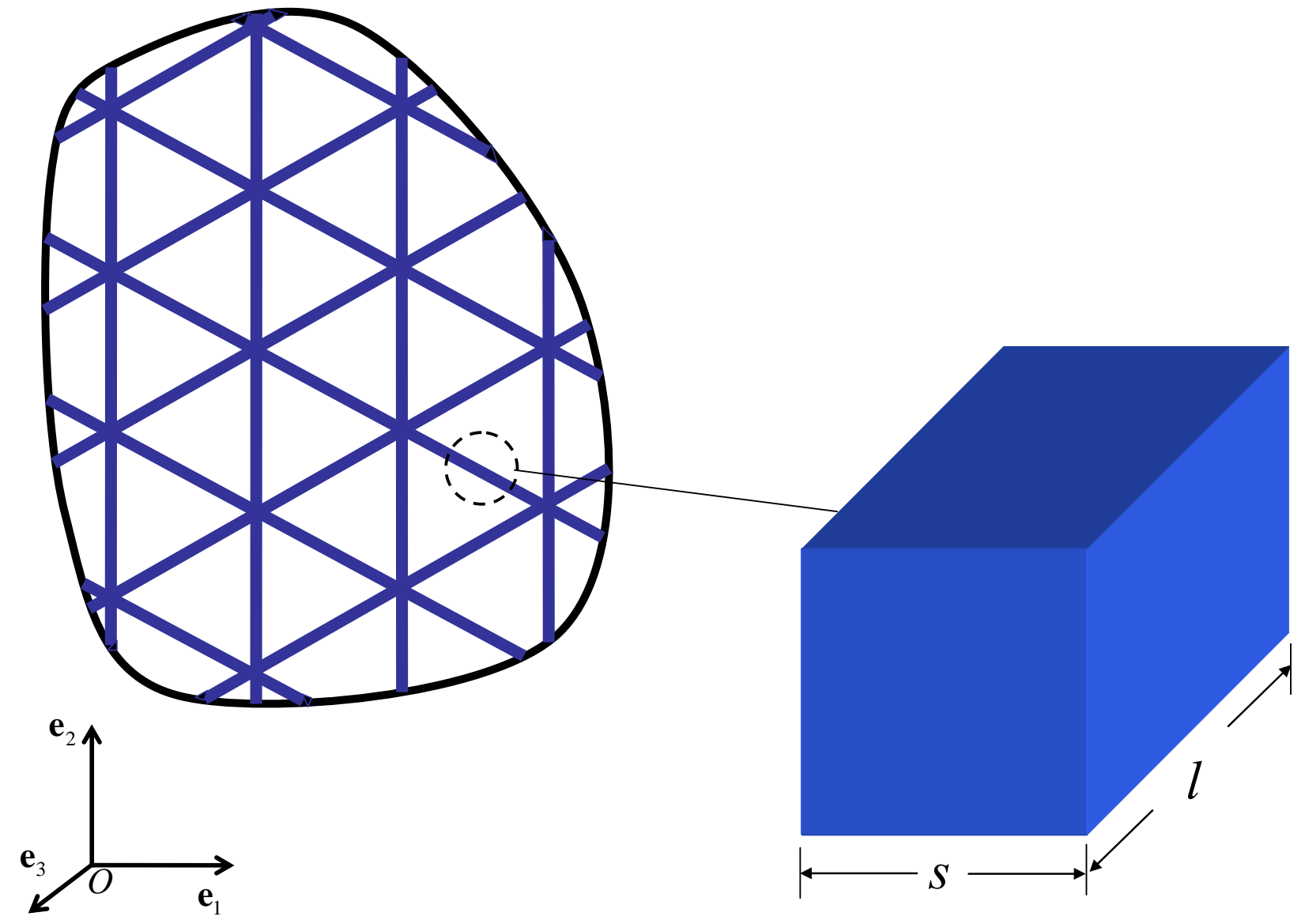

Figure 5 - Triangular lattice strut dimensions 